\title{
Molecular Control of Smooth Muscle Cell Differentiation Marker Genes by Serum Response Factor and Its Interacting Proteins
}

\author{
Tadashi Yoshida \\ Apheresis and Dialysis Center \\ School of Medicine, Keio University \\ Japan
}

\section{Introduction}

Vascular smooth muscle cells (SMCs) exhibit a wide range of different phenotypes at different stages of development (Owens, 1995; Owens et al., 2004; Yoshida \& Owens, 2005). Even in mature animals, SMCs retain the capability to change their phenotype in response to multiple local environmental cues. The plasticity of SMCs enables them to play a critical role in physiological processes in the vasculature, as well as the pathogenesis of numerous vascular diseases including atherosclerosis, re-stenosis after percutaneous coronary intervention, aortic aneurysm, and hypertension. Thus, it is important to understand the precise mechanisms whereby SMCs exhibit different phenotypes under distinct conditions. Because one of the most remarkable differences among SMC subtypes is the difference in expression levels of SMC-specific/-selective genes, elucidation of the molecular mechanisms controlling SMC differentiation marker gene expression may shed light on this issue.

Most of SMC differentiation marker genes characterized to date, including smooth muscle (SM) $\alpha$-actin (Mack \& Owens, 1999), SM-myosin heavy chain (SM-MHC) (Madsen et al., 1998), SM22 $\alpha$ (Li et al., 1996), and h1-calponin (Miano et al., 2000), have multiple highly conserved $\mathrm{CC}(\mathrm{A} / \mathrm{T}-\mathrm{rich})_{6} \mathrm{GG}$ (CArG) elements in their promoter-enhancer regions. Results of studies in vivo have shown that expression of these genes is dependent on the presence of CArG elements (Li et al., 1997; Mack \& Owens, 1999; Manabe \& Owens, 2001a). For example, expression of the SM $\alpha$-actin gene requires a promoter-enhancer region from $-2.6 \mathrm{~kb}$ to +2.8 $\mathrm{kb}$ to recapitulate the expression patterns of the endogenous gene, and mutation of any one of three conserved CArG elements within the regions abolishes the expression (Mack \& Owens, 1999). Likewise, SMC-specific expression of the SM-MHC gene requires $4.2 \mathrm{~kb}$ of the 5 '-flanking region, the entire first exon, and $11.5 \mathrm{~kb}$ of the first intronic sequence, and mutation of CArG elements in the 5 -flanking region abolishes the expression (Manabe \& Owens, 2001a). These results indicate the critical roles of CArG elements in the regulation of SMC differentiation marker gene expression. Currently, it is reported that over 60 of SMCspecific/-selective genes possess CArG elements in the promoter-enhancer regions by insilico analysis (Miano, 2003), although it is not fully determined how many CArG elements of them are functional. 
The binding factor for CArG elements is the ubiquitously expressed transcription factor, serum response factor (SRF) (Norman et al., 1988). Knockout of the SRF gene in mice resulted in early embryonic lethality due to abnormal gastrulation and loss in key mesodermal markers (Arsenian et al., 1998), precluding the evaluation of requirement of SRF for SMC differentiation. Instead, conditional knockout of the SRF gene in the heart and SMCs exhibited the attenuation in cardiac trabeculation and the compact layer expansion, as well as decreases in SMC-specific/-selective genes including SM $\alpha$-actin in aortic SMCs (Miano et al., 2004). Moreover, SRF has been shown to be required for differentiation of SMCs in an in vitro model of coronary SMC differentiation (Landerholm et al., 1999). Indeed, over-expression of dominant-negative forms of SRF inhibited the induction of SMC differentiation marker genes including $S M 22 \alpha$, h1-calponin, and SM $\alpha$-actin in proepicardial cells excised from quail embryos. As such, the preceding studies provide evidence indicating that the CArG-SRF complex plays an important role in the regulation of SMC differentiation marker gene expression. However, SRF was first cloned as a binding factor for the core sequences of serum response element (SRE) in the c-fos gene (Norman et al., 1988). Because the $c-f o s$ gene is known as one of the growth factor-inducible genes, major unresolved issues in the field are to identify the mechanisms whereby: (1) the CArG-SRF complex can simultaneously contribute to two disparate processes: induction of SMC differentiation marker gene expression versus activation of growth-regulated genes; and (2) the ubiquitously expressed SRF can contribute to SMC-specific/-selective expression of target genes.

To date, a number of factors have been reported to interact with SRF. Several recent studies suggest that these interactions are responsible for multiple actions of SRF. Therefore, this review article will summarize recent progress in our understanding of the transcriptional mechanisms involved in controlling expression of SMC differentiation marker genes by focusing on SRF and its interacting factors.

\section{Myocardin is a potent co-factor of SRF for SMC differentiation marker gene expression}

One of the major breakthroughs in the SMC field was the discovery of myocardin (Wang et al., 2001). Myocardin was cloned as a co-factor of SRF by a bioinformatics-based screen and found to be exclusively expressed in SMCs and cardiomyocytes (Chen et al., 2002; Du et al., 2003; Wang et al., 2001; Yoshida et al., 2003). It has two isoforms, and smooth muscleenriched isoform consists of 856 amino acids (Creemers et al., 2006). Myocardin has several domains including three RPEL domains, a basic domain, a glutamine-rich domain, a SAP (Scaffold attachment factors A and B, Acinus, Protein inhibitor of activated STAT) domain, and a leucine zipper-like domain. It has been shown that leucine zipper-like domain is required for homodimerization of myocardin (Figure 1) (Wang et al., 2003), but the function of the other domains is not well understood. Transcriptional activation domain, TAD, is localized at the carboxy-terminal region, and deletion mutants that lack TAD behaved as dominant-negative forms (Wang et al., 2001; Yoshida et al., 2003). Over-expression of myocardin potently induces transcription of virtually all CArG-dependent SMC differentiation marker genes, including SM $\alpha$-actin, SM-MHC, SM22 $\alpha$, h1-calponin, and myosin light chain kinase (MLCK) (Chen et al., 2002; Du et al., 2003; Wang et al., 2001; Wang et al., 2003; Yoshida et al., 2003). Mutation of CArG elements in the SMC promoters abolished 
the responsiveness to myocardin, suggesting that myocardin activates the transcription in a CArG-dependent manner. However, myocardin showed no DNA binding activity, but showed interaction with SRF. In addition, myocardin failed to activate the transcription of CArG-dependent genes in the absence of SRF (Du et al., 2003), demonstrating that myocardin is a co-activator of SRF. Over-expression of myocardin also induced the endogenous expression of SMC differentiation marker genes in cultured SMCs and nonSMCs, including 3T3 fibroblasts, L6 myoblasts, 3T3-L1 preadipocytes, COS cells, and undifferentiated embryonic stem cells (Chen et al., 2002; Du et al., 2003; Du et al., 2004; Wang et al., 2001; Wang et al., 2003; Yoshida et al., 2003; Yoshida et al., 2004b). However, forced expression of myocardin in non-SMCs was not sufficient to induce the full SMC differentiation program, because some SMC-enriched genes, which do not contain CArG elements in their promoter-enhancer region, were not induced (Yoshida et al., 2004b). Nevertheless, it was sufficient to establish a SMC-like contractile phenotype (Long et al., 2008). Either dominant-negative forms of myocardin or siRNA-induced suppression of myocardin decreased the transcription of SMC differentiation marker genes in cultured SMCs (Du et al., 2003; Wang et al., 2003; Yoshida et al., 2003). In addition, myocardindeficient mice exhibited no vascular SMC differentiation and died by embryonic day 10.5 (Li et al., 2003), although this may have been secondary to the defect in the extra-embryonic circulation. Moreover, mice lacking the myocardin gene in neural crest-derived cells died prior to postnatal day 3 from patent ductus arteriosus, and neural crest-derived SMCs in these mice exhibited a cell-autonomous block in expression of SMC differentiation marker genes (Huang et al., 2008). Taken together, the preceding results provide compelling evidence that myocardin plays a key role in the regulation of expression of SMC differentiation marker genes.

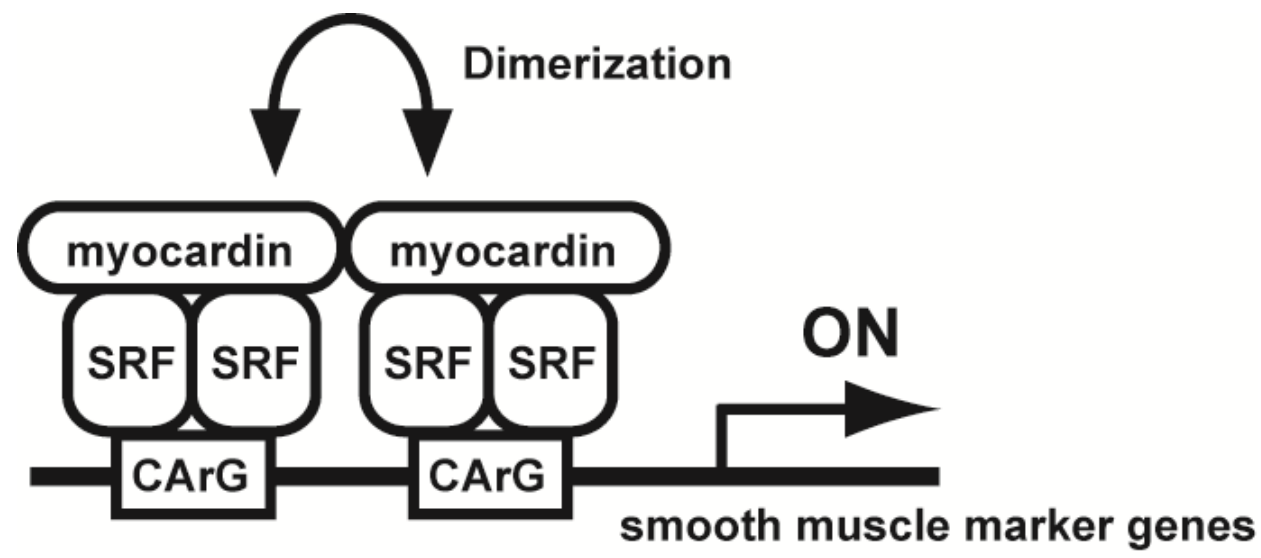

Fig. 1. Myocardin potently induces the transcription of CArG-element containing SMC differentiation marker genes. Myocardin preferentially activates SMC differentiation marker genes which contain multiple CArG elements in their promoter-enhancer regions. Homodimerization of myocardin through the leucine zipper-like domain efficiently activates the transcription. In contrast, myocardin does not induce the transcription of the growth factor-inducible gene, $c-f o s$, because it only contains a single CArG element in the promoter. 


\subsection{Transcriptional mechanism for myocardin-dependent SMC differentiation marker genes}

Although myocardin is a powerful transcriptional co-activator of SRF, there are still some questions for the mechanisms whereby myocardin induces SMC differentiation marker genes. One of these questions is: "what cis-elements and transcriptional co-activators other than SRF are required for the function of myocardin?" Initial studies (Wang et al., 2001) suggested that myocardin activated the transcription through the formation of complex with SRF and multiple CArG elements, based on the findings that: (1) the single CArG-containing c-fos gene had no responsiveness to myocardin; and (2) myocardin could activate an artificial promoter consisting of $4 \times c$-fos SREs coupled to the basal promoter. Such a "2CArG" model, in which multiple CArG elements are required for myocardin-induced transactivation, is strengthened by the results showing that homodimerization of myocardin extraordinary augmented the transcriptional activity of SMC differentiation marker genes (Figure 1) (Wang et al., 2003). However, several SMC-specific genes that only contain single CArG element in their promoter, such as the telokin gene and the cysteine-rich protein-1 (CRP1) gene, have also been shown to be activated by myocardin (Wang et al., 2003; Yoshida et al., 2004b). These results raised a question as to how myocardin distinguishes these single CArG-containing SMC differentiation marker genes from the $c$-fos gene. One hypothesis is that the presence of a ternary complex factor (TCF)-binding site in the c-fos promoter regulates the binding of myocardin to SRF. In support of this, it has been shown that one of the TCFs, Elk-1, could compete for SRF binding with myocardin on the SMC promoters (Wang et al., 2004; Yoshida et al., 2007; Zhou et al., 2005). Such a possibility will be discussed in detail in a later section.

An additional possibility is that degeneracy within CArG elements, i.e. conserved base pair substitutions that reduce SRF binding affinity, contributes to the promoter selectivity of myocardin. Consistent with this idea, the majority of SMC differentiation marker genes including $S M \alpha$-actin and $S M-M H C$ have degenerate CArG elements in their promoterenhancer regions (Miano, 2003). For example, both of CArG elements located within 5'flanking region of the $S M \alpha$-actin gene contain a single $\mathrm{G}$ or $\mathrm{C}$ substitution within their A/Trich cores that is $100 \%$ conserved between species as divergent as humans and chickens (Shimizu et al., 1995). Results of our previous studies showed that substitution of SM $\alpha$-actin 5' CArGs with the c-fos consensus CArGs significantly attenuated injury-induced downregulation of $S M \alpha$-actin expression (Hendrix et al., 2005). In addition, of interest, overexpression of myocardin selectively enhanced SRF binding to degenerate SM $\alpha$-actin CArG elements compared to $c-f o s$ consensus CArG element in SMCs, as determined by quantitative chromatin immunoprecipitation assays. These results raise a possibility that the degeneracy in the CArG elements is one of the determinants of promoter selectivity of myocardin. However, it should be noted that there is a difference not only in the sequence context of CArG elements, but also in the number of CArG elements between the SM $\alpha$-actin gene versus the $c-f o s$ gene. Moreover, there is no $\mathrm{G}$ or $\mathrm{C}$ substitution in the CArG elements of several SMC differentiation marker genes including the SM22 $\alpha$, telokin, and CRP-1 genes (Miano, 2003), although previous studies showed that the binding affinity of SRF to SM22 $\alpha$ CArG-near element was lower than that to the $c$-fos CArG element by electromobility shift assays (EMSA) (Chang et al., 2001). It is interesting to determine whether CArG elements in the telokin gene and the CRP-1 genes also exhibit lower binding affinity to SRF than the $c$-fos 
consensus CArG element. If this is the case, it is likely that reduced SRF binding to CArG elements, which does not necessarily have $\mathrm{G}$ or $\mathrm{C}$ substitutions, is one of the mechanisms for target gene selectivity of myocardin. If this is not the case, it is still possible that the degeneracy in CArG elements may explain a part of the promoter selectivity of myocardin, but this mechanism cannot be applicable to all of the SMC differentiation marker genes.

Regarding the mechanism of myocardin-induced transcription of SMC differentiation marker genes, the physical interaction of myocardin with histone acetyltransferase, p300, and class II histone deacetylases, HDAC4 and HDAC5, has been reported (Cao et al., 2005). Indeed, results showed that over-expression of myocardin induced histone $\mathrm{H} 3$ acetylation in the vicinity of CArG elements at the SM $\alpha$-actin and SM22 $\alpha$ promoters in 10T1/2 cells (Cao et al., 2005). In addition, they showed that p300 augmented the stimulatory effect of myocardin on the transcription of the $S M 22 \alpha$ gene, whereas either HDAC4 or HDAC5 repressed the effect of myocardin by co-transfection/reporter assays. Moreover, they demonstrated that p300 and HDACs, respectively, bound to distinct domains of myocardin simultaneously, suggesting that the balance between p300 and HDACs is likely to be one of the determinants of the transcriptional activity of myocardin.

These results are of significant interest in that they provided evidence that transcription of SMC differentiation marker genes is regulated by the recruitment of chromatin modifying enzymes by myocardin. Previous studies showed that SMC differentiation was associated with increased binding of SRF and hyperacetylation of histones H3 and H4 at CArGcontaining regions of the SM $\alpha$-actin and SM-MHC genes in A404 SMC precursor cells (Manabe \& Owens, 2001b). In addition, we showed that over-expression of myocardin selectively enhanced SRF binding to CArG-containing region of the SM $\alpha$-actin gene, but not to that of the c-fos gene in the context of intact chromatin in SMCs (Hendrix et al., 2005). Results of studies by another group (Qiu \& Li, 2002) also showed that HDACs reduced the transcriptional activity of the SM22 $\alpha$ gene in a CArG-element dependent manner. These findings are consistent with the results showing the association of myocardin with p300 or HDACs (Cao et al., 2005). However, it remains unknown how the association between myocardin and p300 or HDACs regulates the accessibility of SRF to CArG elements, as has been observed during the induction of SMC differentiation in A404 cells (Manabe \& Owens, 2001b). It is possible that particular histone modifications by the myocardin-p300 complex enable SRF to bind to CArG-elements within the SMC promoters. It is also possible that the association between myocardin and chromatin modifying enzymes including p300 may alter the binding affinity of myocardin to SRF. Because regulation of SMC differentiation marker genes by platelet-derived growth factor-BB (PDGF-BB) or oxidized phospholipids has been shown to be accompanied by the recruitment of HDACs and thereby changes in acetylation levels at the SMC promoters (Yoshida et al., 2007, 2008a), it is interesting to determine if these changes are caused by the modulation of association between myocardin and these chromatin modifying enzymes.

\subsection{Role of the myocardin-related family in SMC differentiation}

Two factors were identified as members of the myocardin-related transcription factors: MKL1 (also referred to as MAL, BSAC, and MRTF-A) (Cen et al., 2003; Miralles et al., 2003; Sasazuki et al., 2002; Wang et al., 2002) and MKL2 (also referred to as MRTF-B) (Selvaraj \& Prywes, 2003; Wang et al., 2002). It has been shown that expression of MKL1 mRNA is 
ubiquitous, whereas expression of $M K L 2$ mRNA is restricted to several tissues including the brain and the heart (Cen et al., 2003; Selvaraj \& Prywes, 2003; Wang et al., 2002). Cotransfection studies revealed that both MKL1 and MKL2 were capable of inducing the transcription of multiple CArG-containing promoters including atrial natriuretic factor (ANF), SM22 $\alpha, S M \alpha$-actin, and cardiac $\alpha$-actin. A truncated MKL2 protein that lacks both aminoterminal region and carboxy-terminal region (MKL2 $\Delta \mathrm{N} \Delta \mathrm{C} 700$ ) behaved as a dominantnegative manner for both MKL1 and MKL2, and over-expression of MKL2 $\Delta \mathrm{N} \Delta \mathrm{C} 700$ inhibited skeletal muscle differentiation in C2C12 skeletal myoblasts (Selvaraj \& Prywes, 2003). In addition, MKL1 strongly induced SMC differentiation marker gene expression in undifferentiated embryonic stem cells, even in the absence of myocardin (Du et al., 2004). Moreover, a truncated form of MKL1, which behaved as a dominant-negative form of MKL1 and myocardin, inhibited MKL1-induced transcription of the SM22 $\alpha$ gene (Du et al., 2004). Taken together, MKL factors appear to be important regulators of SMC differentiation marker gene expression as well as myocardin, and they appear to exhibit the redundant function with myocardin as SRF co-factors. However, the precise roles of MKL factors in SMC differentiation marker gene expression in SMCs are still unclear, because most of these studies analyzing the function of MKL factors have been performed by over-expression experiments. Regarding this point, there are several interesting studies as described below. First, MKL1 knockout mice were viable, but were unable to effectively nurse their offspring due to a failure in maintenance of the differentiated state of mammary myoepithelial cells during lactation ( $\mathrm{Li}$ et al., 2006; Sun et al., 2006). Second, conditional knockout of the MKL2 gene in neural crestderived cells exhibited a spectrum of cardiovascular defects including abnormal patterning of the branchial arch arteries (Li et al., 2005; Oh et al., 2005). The abnormalities in MKL2 knockout mice were accompanied by a decrease in SM $\alpha$-actin expression in SMCs within the branchial arch arteries. Based on the results of these studies, MKL1 is unlikely to play an important role in expression of SMC differentiation marker genes in vivo. In addition, role of MKL2 for SMC differentiation in SMCs derived from other origins is still unknown. A biggest issue is how broadly expressed MKL factors regulate SMC-specific/-selective CArG-dependent genes. Recently, several studies suggest the importance of intracellular localization of MKL factors in SMCs and non-SMCs (Hinson et al., 2007; Nakamura et al., 2010; Yoshida et al., 2007). Further studies are required to address this issue.

In summary, it is clear that myocardin plays a critical role in SMC differentiation in concert with the CArG-SRF complex. However, myocardin is not a SMC-specific gene in that it is also expressed in cardiomyocytes, suggesting that myocardin alone is not enough to coordinate expression of SMC differentiation marker genes. It is highly likely that cooperative interaction of the SRF-myocardin complex with other transcription factors is necessary for expression of SMC differentiation marker genes in SMCs. Further studies are needed to clarify these combinatorial mechanisms.

\section{Ternary complex factors exhibit dual roles in the transcription of SRF- dependent CArG-Containing genes}

TCFs are a subfamily of the Ets domain transcription factors (Buchwalter et al., 2004). TCF was first described as $62 \mathrm{kD}$ nuclear fractions (p62) that form a ternary complex with SRF on the $c$-fos SRE (Shaw et al., 1989). Three members, Elk-1, Sap-1/Elk-4, and Net/Sap-2/Elk-3, have been identified as TCFs. Previous studies demonstrated that TCFs are present on SREs 
of the $c$-fos gene with SRF dimers both before and after growth factor stimulation, and that after the stimulation with growth factors, TCFs are phosphorylated and activate transcription of the $c-f o s$ gene (Buchwalter et al., 2004).

Although it has been believed, for a long time, that most of SMC differentiation marker genes lack the TCF-binding site in their promoter regions (Miano 2003), results of recent studies by multiple laboratories including our own (Wang et al., 2004; Yoshida et al., 2007; Zhou et al., 2005) suggest the involvement of Elk-1 in the regulation of SMC differentiation marker genes. They presented evidence that repression of SMC differentiation marker genes including SM $\alpha$-actin and SM22 $\alpha$ by PDGF-BB was due to the displacement of myocardin from SRF by phosphorylated Elk-1 in cultured SMCs (Figure 2). Indeed, they showed that treatment with PDGF-BB induced phosphorylation of Elk-1 through the activation of the MEK1/2-Erk1/2 pathway and increased the association between Elk-1 and SRF, whereas the association between myocardin and SRF was decreased at the same time. By extensively mapping the domain of myocardin and Elk-1, they found that both factors have a structurally related SRF-binding motif and thereby compete for the common docking region of SRF. These results are very interesting in that phosphorylation of Elk-1 simultaneously exhibits the dual roles in the regulation of CArG-dependent genes: transcriptional activation of the $c$-fos gene versus transcriptional repression of SMC differentiation marker genes.

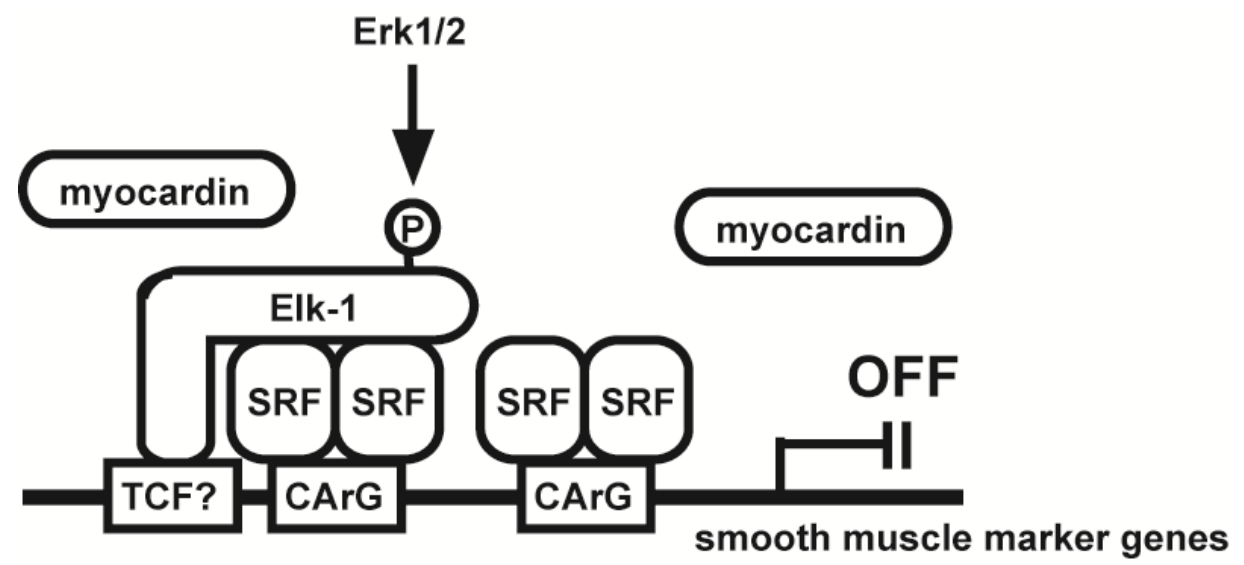

Fig. 2. Phosphorylation of Elk-1 competes for SRF binding with myocardin. The myocardinSRF-CArG complex activates the transcription of SMC differentiation marker genes in the absence of growth factors as shown in Fig. 1. Activation of the Erk1/2 pathway by growth factors such as PDGF-BB induces phosphorylation of Elk-1. Phosphorylated Elk-1 displaces myocardin from SRF and binds to SRF, thereby suppressing the transcription of SMC differentiation marker genes. It has been reported that phosphorylated Elk-1 is able to bind to the TCF-binding site within the SM22 $\alpha$ promoter (Wang et al., 2004), although the TCFbinding site is not present within the promoter region of most SMC differentiation marker genes.

However, the mechanisms responsible for these dual effects have not been clearly understood yet. That is, although the binding of Elk- 1 on the putative TCF-binding site (5'TTCCCG-3') adjacent to the CArG-far element at the SM22 $\alpha$ promoter was detected by 
EMSA and chromatin immunoprecipitation assays (Wang et al., 2004), this sequence is not the consensus binding site for Elk-1 (Treisman et al., 1992). By using "the site selection method" to purify DNA capable of forming ternary complexes from a pool of randomized oligonucleotides, the consensus binding motif for Elk-1 and Sap-1 was determined as $5^{\prime}$ (C/A)(C/A)GGA(A/T)-3' previously (Treisman et al., 1992). The putative TCF-binding site within the SM22 $\alpha$ gene (sense: 5'-TTCCCG-3' and antisense: 5'-CGGGAA-3') does not match this sequence completely. In addition, although over-expression of Elk-1 downregulated the $S M 22 \alpha$ promoter-luciferase activity through the competition with myocardin, this competition was still observed when the mutational SM22 $\alpha$-luciferase construct, in which the putative TCF-binding site was abolished, was used. Furthermore, there is no putative Elk-1 binding site near the CArG elements within the SM $\alpha$-actin promoter (Mack \& Owens, 1999). Because chromatin immunoprecipitation assays can detect not only the direct binding of protein to DNA sequence, but also the binding of protein to protein, it is highly possible that the attachment of Elk-1 to the TCF-binding site may not be absolutely required for the competition with myocardin for SRF binding. Nevertheless, the $S M 22 \alpha$ promoter with a mutation in the TCF-binding site has been reported to direct ectopic transcription in the heart in a later embryonic stage, as compared with the wild-type SM22 $\alpha$ promoter in vivo (Wang et al., 2004). Further studies are needed to determine if these findings are applicable to multiple SMC differentiation marker genes.

It is also of interest to determine whether the activation of Elk-1 can recruit histone deacetylases to the promoter regions of SMC differentiation marker genes. Elk-1 contains two transcriptional repression domains, an $\mathrm{N}$-terminal transcriptional repression domain and an $\mathrm{R}$ motif located in the C-terminal transcriptional activation domain (Buchwalter et al., 2004). It has been shown that HDAC1 and HDAC2 were recruited to the N-terminal transcriptional repression domain of Elk- 1 on the $c-f o s$ promoter followed by the activation of the MEK1/2-Erk-1/2 pathway, and this recruitment kinetically correlated with the shutoff of the $c$-fos gene expression after growth factor stimulation (Yang et al., 2001; Yang \& Sharrocks, 2004). We previously showed that repression of SMC differentiation marker genes after stimulation with PDGF-BB was accompanied by the recruitment of multiple HDACs, HDAC2, HDAC4, and HDAC5 in cultured SMCs (Yoshida et al., 2007). It is possible that the association between Elk-1 and these HDACs on the SMC promoters is one of the mechanisms for repression of SMC differentiation marker gene expression. Moreover, it was reported that SUMO modification of the $\mathrm{R}$ motif in Elk-1 could antagonize the MEK1/2-Erk1/2 pathway and repress the transcription of the $c$-fos gene (Yang et al., 2003). Thus, it is also possible that PDGF-BB can induce sumoylation of Elk-1 and exhibit the repressive effects on SMC differentiation marker genes.

In summary, the preceding results indicate that Elk-1 plays dual roles in the transcription of CArG-dependent genes as both an activator and a repressor. However, there are still some questions as discussed above. Clearly, one of the most fascinating questions is to determine if knockdown of Elk-1 abolishes PDGF-BB-induced repression of SMC differentiation marker genes both in vivo and in vitro.

\section{Multiple homeodomain proteins regulate SMC differentiation}

Homeodomain proteins are a family of transcription factors with a highly conserved DNAbinding domain that regulate cell proliferation, differentiation, and migration in many cell 
types during embryogenesis (Gorski \& Walsh, 2003). This family is comprised of over 160 genes, and it has been reported that several homeodomain proteins are able to regulate differentiation of SMCs by interacting with the CArG-SRF complex.

One of these factors is Prx-1 (Paired-related homeobox gene-1), which is also known as MHox and Phox (Cserjesi et al., 1992; Grueneberg et al., 1992). Expression of Prx-1 is completely restricted to mesodermally derived cell types during embryogenesis and to cell lines of mesodermal origin including cultured aortic SMCs (Blank et al., 1995; Cserjesi et al., 1992). Previous studies from our laboratory and others showed that Prx- 1 was capable of inducing the transcription of the CArG-SRF dependent genes (Grueneberg et al., 1992; Hautmann et al., 1997; Yoshida et al., 2004a). Indeed, we found that angiotensin II increased expression of multiple SMC differentiation marker genes including SM $\alpha$-actin, as well as Prx-1 expression in cultured SMCs (Hautmann et al., 1997; Turla et al., 1991; Yoshida et al., 2004a). Of major interest, we provided evidence that siRNA-induced suppression of Prx-1 dramatically reduced both basal and angiotensin II-induced transcription of the SM $\alpha$-actin gene (Yoshida et al., 2004a). In addition, Prx-1 increased the SRF binding to degenerate CArG B element within the SM $\alpha$-actin gene by EMSA (Hautmann et al., 1997). Similarly, Prx-1 enhanced the binding of SRF to $c$-fos CArG element by EMSA (Grueneberg et al., 1992). However, the formation of a stable higher order complex comprised of Prx-1, SRF, and CArG element was not detected by EMSA. Rather, Prx-1 enhanced both the rate of association and the rate of dissociation between SRF and CArG element, thereby increasing the rate of exchange of SRF on the CArG element. Although further studies are required to clarify these mechanisms in detail, results thus far suggest that Prx-1 plays a key role in the transcription of CArG-dependent genes through regulating the binding of SRF to CArG elements.

Although the preceding results suggest that Prx-1 is involved in the regulation of SMC differentiation marker gene expression (Hautmann et al., 1997; Yoshida et al., 2004a), it also plays a role in proliferation of SMCs. Prx-1 expression was induced during the development of pulmonary vascular disease in adult rats, and Prx-1 enhanced the proliferation rate of cultured rat A10 SMCs via the induction of tenascin-C expression (Jones et al., 2001). Taken together, results suggest that Prx-1 plays multiple roles in the regulation of differentiation status and the regulation of proliferation status in SMCs. This is consistent with the idea that differentiation and proliferation are not necessarily mutually exclusive processes (Owens \& Thompson, 1986; Owens et al., 2004). However, it remains unknown whether Prx-1 exhibits these two roles simultaneously or Prx-1 exhibits distinct roles in a developmental stagespecific manner. Of interest, Prx -1 knockout mice have been made and shown to exhibit major defects in skeletogenesis and die soon after birth (Martin et al., 1995). Mice null for both Prx-1 and its homologue, Prx-2, showed a vascular abnormality with an abnormal positioning and awkward curvature of the aortic arch and a misdirected and elongated ductus arteriosus (Bergwerff et al., 2000). Moreover, expression of endothelial markers such as Flk-1 and VCAM-1 and von Willebrand factor-positive cells were decreased in the lung of Prx-1 null newborn mice (Ihida-Stansbury et al., 2004), suggesting that Prx-1 is required for lung vascularization in vivo. It will be of interest to directly test the role of Prx-1 in CArGdependent SMC differentiation marker gene expression in these mice.

Another homeodomain protein related to SMC differentiation is Hex. Hex was originally isolated from hematopoietic tissues by PCR using degenerate oligonucleotide primers corresponding to the conserved homeodomain sequences and has been shown to play an 
important role in inducing differentiation of vascular endothelial cells (Thomas et al., 1998). In SMCs, Hex protein expression was induced in the neointima after balloon injury of rat aorta, while it was undetectable in normal aorta (Sekiguchi et al., 2001). The expression pattern of Hex was similar to that of SMemb/NMHC-B, a marker of phenotypically modulated SMCs. Hex induced the transcription of the SMemb promoter, and cAMPresponsive element (CRE) located at $-481 \mathrm{bp}$ within the promoter was critical for Hex responsiveness. However, Hex failed to bind to CRE directly, thus the precise mechanisms whereby Hex activated the $S M e m b$ promoter are still unclear. Of interest, subsequent studies showed that Hex also induced expression of a subset of SMC differentiation marker genes including SM $\alpha$-actin and SM22 $\alpha$, but not SM-MHC and h1-calponin (Oyama et al., 2004). Hex induced the transcription of the SM22 $\alpha$ gene in a CArG-dependent manner, and it enhanced the binding of SRF to CArG-near element within the SM22 $\alpha$ promoter, as determined by EMSA. In addition, immunoprecipitation assays revealed the physical association between SRF and Hex. As such, the mechanisms whereby Hex induces SMC differentiation marker genes seem to be similar to those of Prx-1. However, results showing that Hex simultaneously activated expression of both SMC differentiation marker genes and those characteristic of phenotypically modulated SMCs are paradoxical, and further studies are clearly needed to precisely define the pathophysiological role of Hex in SMCs.

Nkx-3.2 is also a homeodomain protein that regulates expression of SMC differentiation marker genes (Nishida et al., 2002). It has been demonstrated that a triad of SRF, GATA-6, and Nkx-3.2 formed a complex with their corresponding cis-elements and cooperatively transactivated SMC differentiation marker genes including $\alpha 1$-integrin, $S M 22 \alpha$, and caldesmon. Because co-localization of GATA-6, Nkx-3.2, and SRF was exclusively observed in SMCs, SMC-specific gene expression does not appear to be the result of any single transcription factor that is unique to SMCs, but rather is due to unique combinatorial interactions of factors that may be expressed in multiple cell types but only found together in SMCs.

Furthermore, we recently identified Pitx2 as a homeodomain protein which is required for the initial induction of SMC differentiation by using a subtraction hybridization screen (Shang et al., 2008). Over-expression of Pitx2 induced expression of CArG-dependent SMC differentiation marker genes, whereas knockdown of Pitx2 attenuated retinoic acid-induced differentiation of SMCs from undifferentiated SMC precursor cells. Furthermore, Pitx2 knockout mouse embryos exhibited impaired induction of SMC differentiation markers in the dorsal aorta and branchial arch arteries. We identified three mechanisms for Pitx2induced transcription of SMC differentiation marker genes (Figure 3). First, Pitx2 bound to its consensus TAATC $(\mathrm{C} / \mathrm{T})$ element in the promoter region of SMC differentiation marker genes. Second, Pitx2 physically associated with SRF. Third, Pitx2 mediated exchange of HDACs with p300 to increase acetylation levels of histone $\mathrm{H} 4$ at the SMC promoters. These results provide compelling evidence that Pitx 2 plays a critical role in the induction of SMC differentiation during the early embryogenesis. Further studies are needed to determine if Pitx2 also contributes to the pathogenesis of vascular diseases including atherosclerosis.

As such, several homeodomain proteins are involved in the regulation of CArG-SRF dependent SMC differentiation marker gene expression, and some of the mechanisms appear to be mediated by common pathways. Further studies are needed to clarify the temporal and spatial roles of each of these homeodomain proteins in SMC differentiation. 


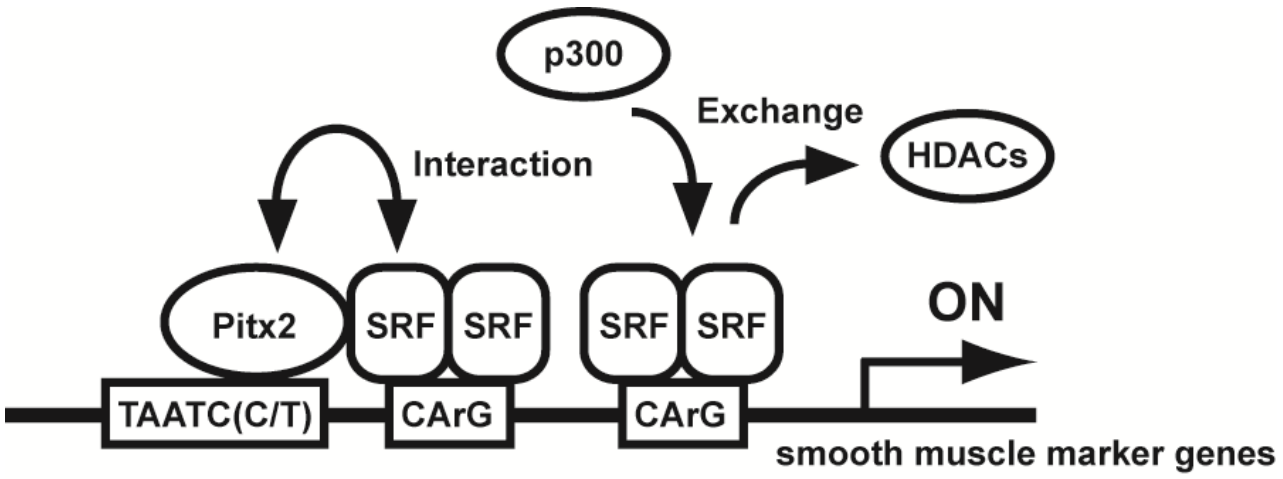

Fig. 3. Pitx2 transactivates SMC differentiation marker genes through three mechanisms. Pitx2 induces expression of SMC differentiation marker genes by: (1) binding to a consensus TAATC(C/T) cis-element; (2) interacting with SRF; and (3) mediating exchange of HDACs with p300 at the promoter region of SMC differentiation marker genes. These mechanisms are important for the initial induction of SMC differentiation during the early embryonic development.

\section{A number of factors associate with SRF}

In addition to the factors described above, there are a number of transcription factors known to interact with SRF. These factors also play key roles in the control of SMC differentiation marker gene expression. In this section, some of these transcription factors will be discussed briefly.

\subsection{GATA-6}

GATA proteins are a family of zinc finger transcription factors, and play essential roles in development through their interaction with a DNA consensus element, "WGATAR" (Molkentin, 2000). Six GATA transcription factors have been identified in vertebrates, and GATA-4, GATA-5, and GATA-6 are thought to be involved in the formation of the heart, gut, and vessels. During the early murine embryonic development, expression patterns of GATA-6 and GATA-4 were similar, with expression being detected in the precardiac mesoderm, the embryonic heart tube, and the primitive gut (Morrisey et al., 1996). However, during the late development, GATA- 6 became the only GATA factor to be expressed in vascular SMCs. Knockout of the GATA- 6 gene in mice resulted in embryonic lethality between embryonic day 6.5 and 7.5, precluding the evaluation of the role of GATA-6 in SMC differentiation and maturation (Morrisey et al., 1998).

As described in a previous section, GATA-6 has shown to interact with SRF and Nkx-3.2 and to induce SMC differentiation marker gene expression (Morrisey et al., 1998; Nishida et al., 2002). GATA-6 expression in SMCs was rapidly downregulated after vascular injury in rat carotid arteries, and adenovirus-mediated transfer of GATA- 6 to the vessel wall after the balloon injury partially inhibited the formation of intimal thickening and reversed the downregulation of SMC differentiation marker genes including SM $\alpha$-actin and SM-MHC (Mano et al., 1999). These results suggest the important role of GATA-6 in regulating SMC 
differentiation. Of interest, results of studies (Yin \& Herring, 2005) showed that GATA-6 increased the transcriptional activity of the SM $\alpha$-actin and SM-MHC genes, whereas it reduced the transcriptional activity of the telokin gene. They found that the GATA- 6 binding site was located adjacent to CArG element in the telokin promoter and that over-expression of GATA-6 interfered the interaction between myocardin and SRF by mammalian twohybrid assays. However, it is unclear why GATA-6 has positive and negative effects on CArG-dependent SMC differentiation marker genes. It is possible that these opposite effects are due to the number of CArG elements or the distance between the GATA- 6 binding site and the CArG element. Further studies are needed to test these possibilities.

\subsection{KIf4}

Klf4 is a member of Krüppel-like transcriptional factors that have recently received increased attention. Previously, Klf4 was identified as a binding factor for the transforming growth factor- $\beta 1$ control element (TCE) found in the promoter region of the SM $\alpha$-actin and SM22 $\alpha$ genes, based on a yeast one-hybrid screen (Adam et al., 2000). Klf4 exhibited a profound inhibitory effect on expression of SMC differentiation marker genes via a TCEdependent and a CArG-SRF-dependent manner (Liu et al., 2003, 2005). For example, adenovirus-mediated over-expression of Klf4 repressed endogenous expression of SM $\alpha$ actin and SM-MHC genes, as well as expression of myocardin, in cultured SMCs as measured by real-time reverse transcription-PCR (Liu et al., 2005). In addition, over-expression of Klf4 completely abolished myocardin-induced activation of SMC differentiation marker genes. Co-immunoprecipitation assays revealed that Klf4 physically interacted with SRF, and chromatin immunoprecipitation assays showed that over-expression of Klf4 markedly reduced the binding of SRF to CArG elements on the SM $\alpha$-actin promoter in intact chromatin of cultured SMCs (Liu et al., 2005). Moreover, PDGF-BB treatment induced Klf4 mRNA expression in cultured SMCs, and siRNA-induced suppression of Klf4 partially blocked PDGF-BB-induced suppression of SMC differentiation marker genes (Liu et al., 2005). Of significant interest, we demonstrated that conditional knockout of the Klf4 gene in mice exhibited a delay in suppression of SMC differentiation markers, and an enhanced neointimal formation following vascular injury (Figure 4) (Yoshida et al., 2008b). Additionally, we showed that Klf4, Elk-1, and HDACs cooperatively suppress oxidized phospholipid-induced suppression of SMC differentiation marker genes in cultured SMCs (Yoshida et al., 2008a). Taken together, these results suggest that Klf4 plays a key role in mediating phenotypic switching of SMCs.

\subsection{Cysteine-rich LIM-only proteins, CRP1 and CRP2}

The members of the cysteine-rich LIM-only protein (CRP) family, CRP1 and CRP2, are expressed predominantly in SMCs and contain two LIM domains in the structure (Henderson et al., 1999; Jain et al., 1996). It is known that the functions of LIM domains are to mediate protein-protein interactions, to target proteins to distinct subcellular locations, and to mediate assembly of multimeric protein complexes. One of the functions of CRP1 and CRP2 is to interact with both the actin crosslinking protein, $\alpha$-actinin, and the adhesion plaque protein, zyxin, and to regulate the stability and structure of adhesion complexes (Arber \& Caroni, 1996; Schmeichel \& Beckerle, 1994). In addition to such a cytoplasmic role, 

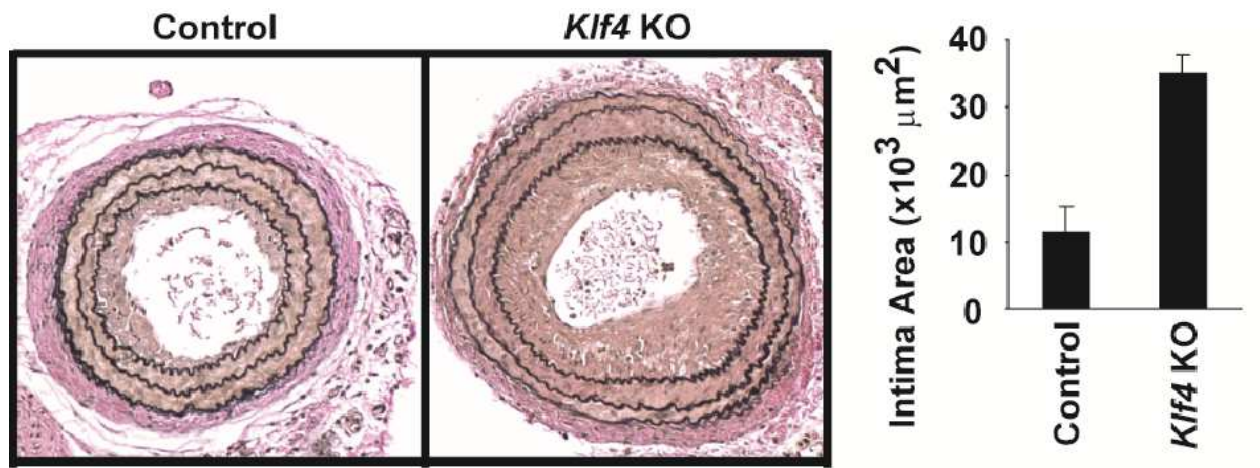

Fig. 4. Conditional knockout of the Klf4 gene in mice accelerates neointimal formation following vascular injury. Klf4 is a potent repressor of SMC differentiation marker genes. Interestingly, conditional knockout of the Klf4 gene in mice delays downregulation of SMC differentiation markers, but also accelerates neointimal formation after vascular injury (Yoshida et al., 2008).

it has been reported that CRP1 and CRP2 are also able to function as transcriptional cofactors (Chang et al., 2003). Over-expression of three factors, SRF, GATA-6, and CRP1/CRP2 strongly activated the transcription of SMC differentiation marker genes including SM $\alpha$ actin, SM-MHC, SM22 $\alpha$, h1-calponin, and h-caldesmon. The N-terminal LIM domain of CRP1/2 interacted with SRF, and that the C-terminal LIM domain of CRP1/2 interacted with GATA-6, and that SRF and GATA-6 also interacted each other. These results suggest a critical role of CRP1/2 in organizing multiprotein complexes onto the SMC promoters for SMC differentiation. However, it is still unclear how CRP1 and CRP2 are translocated from the cytoplasm to the nucleus and what signaling pathways control their nuclear localization. Moreover, there is a lack of evidence that these factors play a role in control of SMC differentiation marker gene expression in vivo in SMCs. Indeed, results of recent studies showed that SMC differentiation in CRP1 knockout mice or CRP2 knockout mice appeared to be normal, although neointimal formation was altered after vascular injury (Lilly et al., 2010; Wei et al., 2005). Results raised a question as to the role of CRP1/2 in SMC differentiation.

\subsection{PIAS-1}

Results of previous studies showed that over-expression of class I basic Helix-Loop-Helix proteins, E2-2, and SRF exhibited a synergistic effect on the transcription of the SM $\alpha$-actin promoter-enhancer in BALBc/3T3 cells (Kumar et al., 2003). However, direct interaction between E2-2 and SRF was undetectable by EMSA using the recombinant proteins. We isolated PIAS-1 (protein inhibitor of activated STAT-1) as an interacting protein for E2-2 by a yeast two-hybrid screen (Kawai-Kowase et al., 2005). We also found that PIAS-1 interacted with SRF, suggesting that PIAS-1 works as a bridging molecule between E2-2 and SRF. Interestingly, PIAS-1 belongs to a family of E3 ligases which promote SUMO modifications of target proteins (Schmidt \& Müller, 2002). Indeed, recent studies showed that transcription factors involved in SMC differentiation, such as myocardin and Klf4, were sumoylation 
targets of PIAS-1. Myocardin sumoylation by PIAS-1 transactivated cardiogenic genes in 10T1/2 fibroblasts (Wang et al., 2007), whereas sumoylation of Klf4 by PIAS-1 promoted transforming growth factor- $\beta$ induced activation of SM $\alpha$-actin expression in SMCs (KawaiKowase et al., 2009). Further studies are needed to determine effects of PIAS-1 knockout on SMC differentiation as well as phenotypic switching of SMCs.

\section{Conclusion and perspectives}

As discussed above, it is clear that the CArG-SRF complex plays a central role in the regulation of SMC differentiation marker gene expression. However, it is also clear that expression of SMC differentiation marker genes is not controlled by the CArG-SRF complex alone, nor by any single transcription factor that is expressed exclusively in SMCs. Rather, SMC-selective gene expression appears to be mediated by complex combinatorial interactions of multiple transcription factors and co-factors, including some that are ubiquitously expressed like SRF and PIAS-1, as well as others that are selective for SMCs like myocardin, Prx-1, CRP-1/2, and GATA-6. In addition to the transcription factors described above, several novel factors, including Fhl2 (Philippar et al., 2004), HERP1 (Doi et al., 2005) and lupaxin (Sundberg-Smith et al., 2008), have also been identified as factors interacting with SRF.

However, our knowledge is immature regarding the overall connection among multiple transcription factors and co-factors that can modify the activity of SRF. Most of studies analyzing the protein-protein interaction thus far have been focused on the relationship among two or three proteins. However, a number of factors should be coordinately regulated and interacted by a single environmental cue. It is of interest to determine whether all of SRF-interacting factors are simultaneously required for SMC differentiation marker gene expression or these factors independently contribute to SMC differentiation marker gene expression in time- and position-specific manner. Thus, in the long term, future studies in the SMC field are needed not only to screen out other key transcription factors, but also to map out the connection networks of these factors.

During the past decade, there is a tremendous progress in our understanding of the roles of chromatin modifying enzymes and chromatin structure in gene transcription in all cell types. Accumulating evidence indicates that the $\mathrm{N}$-terminal tails of histones are the target of numerous modifications, including acetylation, methylation, phosphorylation, ubiqutination, and ADP ribosylation, and that these modifications control gene transcription (Fischle et al., 2003). However, this issue in the SMC field is obviously in its infancy. Thus far, only several transcription factors have been reported to be involved in chromatin remodeling. Clearly, more detailed studies are required to determine the mechanisms whereby SRF and its interacting factors coordinately contribute to chromatin remodeling.

Finally, although much progress has been made in our understanding of the role of transcription factors in the control of SMC differentiation marker gene expression, some of these studies are performed only in cultured SMCs or SM-like systems. Studies of these factors in vivo will provide more compelling information to enhance our knowledge about SMC differentiation and development. 


\section{Acknowledgments}

This work was supported in part by Keio Gijuku Academic Development Funds and Takeda Science Foundation.

\section{References}

Adam, P.J.; Regan, C.P.; Hautmann, M.B. \& Owens, G.K. (2000) Positive- and negativeacting Krüppel-like transcription factors bind a transforming growth factor $\beta$ control element required for expression of the smooth muscle cell differentiation marker SM22 $\alpha$ in vivo. J Biol Chem. 275:37798-37806

Arber, S. \& Caroni, P. (1996) Specificity of single LIM motifs in targeting and LIM/LIM interactions in situ. Genes Dev. 10:289-300

Arsenian, S.; Weinhold, B.; Oelgeschläger, M.; Rüther, U. \& Nordheim, A. (1998) Serum response factor is essential for mesoderm formation during mouse embryogenesis. EMBO J. 17:6289-6299

Bergwerff, M.; Gittenberger-de Groot, A.C.; Wisse, L.J.; DeRuiter, M.C.; Wessels, A.; Martin, J.F. et al. (2000) Loss of function of the Prx1 and Prx2 homeobox genes alters architecture of the great elastic arteries and ductus arteriosus. Virchows Arch. 436:12-19

Blank, R.S.; Swartz, E.A.; Thompson, M.M.; Olson, E.N. \& Owens, G.K. (1995) A retinoic acid-induced clonal cell line derived from multipotential P19 embryonal carcinoma cells expresses smooth muscle characteristics. Circ Res. 76:742-749

Buchwalter, G.; Gross, C. \& Wasylyk, B. (2004) Ets ternary complex transcription factors. Gene. 324:1-14

Cao, D.; Wang, Z.; Zhang, C.L.; Oh, J.; Xing, W.; Li, S. et al. (2005) Modulation of smooth muscle gene expression by association of histone acetyltransferases and deacetylases with myocardin. Mol Cell Biol. 25:364-376

Cen, B.; Selvaraj, A.; Burgess, R.C.; Hitzler, J.K.; Ma, Z.; Morris, S.W. et al. (2003) Megakaryoblastic leukemia 1, a potent transcriptional coactivator for serum response factor (SRF), is required for serum induction of SRF target genes. Mol Cell Biol. 23:6597-6608

Chang, D.F.; Belaguli, N.S.; Iyer, D.; Roberts, W.B.; Wu, S.P.; Dong, X.R. et al. (2003) Cysteine-rich LIM-only proteins CRP1 and CRP2 are potent smooth muscle differentiation cofactors. Dev Cell. 4:107-118

Chang, P.S.; Li, L.; McAnally, J. \& Olson, E.N. (2001) Muscle specificity encoded by specific serum response factor-binding sites. J Biol Chem. 276:17206-17212

Chen, J.; Kitchen, C.M.; Streb, J.W. \& Miano, J.M. (2002) Myocardin: a component of a molecular switch for smooth muscle differentiation. J Mol Cell Cardiol. 34:1345-1356

Creemers, E.E.; Sutherland, L.B.; Oh, J.; Barbosa, A.C. \& Olson, E.N. (2006) Coactivation of MEF2 by the SAP domain proteins myocardin and MASTR. Mol Cell. 23:83-96

Cserjesi, P.; Lilly, B.; Bryson, L.; Wang, Y.; Sassoon, D.A. \& Olson, E.N. (1992) MHox: a mesodermally restricted homeodomain protein that binds an essential site in the muscle creatine kinase enhancer. Development. 115:1087-1101

Doi, H.; Iso, T.; Yamazaki, M.; Akiyama, H.; Kanai, H.; Sato, H. et al. (2005) HERP1 inhibits myocardin-induced vascular smooth muscle cell differentiation by interering with SRF binding to CArG box. Arterioscler Thromb Vasc Biol. 25:2328-2334 
Du, K.L.; Ip, H.S.; Li, J.; Chen, M.; Dandre, F.; Yu, W. et al. (2003) Myocardin is a critical serum response factor cofactor in the transcriptional program regulating smooth muscle cell differentiation. Mol Cell Biol. 23:2425-2437

Du, K.L.; Chen, M.; Li, J.; Lepore, J.J.; Mericko, P. \& Parmacek, M.S. (2004) Megakaryoblastic leukemia factor-1 transduces cytoskeletal signals and induces smooth muscle cell differentiation from undifferentiated embryonic stem cells. J Biol Chem. 279:1757817586

Fischle, W.; Wang, Y. \& Allis, C.D. (2003) Histone and chromatin cross-talk. Curr Opin Cell Biol. 15:172-183

Gorski, D.H. \& Walsh, K. (2003) Control of vascular cell differentiation by homeobox transcription factors. Trends Cardiovasc Med. 13:213-220

Grueneberg, D.A.; Natesan, S.; Alexandre, C. \& Gilman, M.Z. (1992) Human and Drosophila homeodomain proteins that enhance the DNA-binding activity of serum response factor. Science. 257:1089-1095

Hautmann, M.B.; Thompson, M.M.; Swartz, E.A.; Olson, E.N. \& Owens, G.K. (1997) Angiotensin II-induced stimulation of smooth muscle $\alpha$-actin expression by serum response factor and the homeodomain transcription factor MHox. Circ Res. 81:600-610

Henderson, J.R.; Macalma, T.; Brown, D.; Richardson, J.A.; Olson, E.N. \& Beckerle, M.C. (1999) The LIM protein, CRP1, is a smooth muscle marker. Dev Dyn. 214:229-238

Hendrix, J.A.; Wamhoff, B.R.; McDonald, O.G.; Sinha, S.; Yoshida, T. \& Owens, G.K. (2005) $5^{\prime}$ CArG degeneracy in smooth muscle $\alpha$-actin is required for injury-induced gene suppression in vivo. J Clin Invest. 115:418-427

Hinson, J.S.; Medlin, M.D.; Lockman, K.; Taylor, J.M. \& Mack, C.P. (2007) Smooth muscle cell-specific transcription is regulated by nuclear localization of the myocardinrelated transcription factors. Am J Physiol Heart Circ Physiol. 292:H1170-H1180

Huang, J.; Cheng, L.; Li, J.; Chen, M.; Zhou, D.; Lu, M.M. et al. (2008) Myocardin regulates expression of contractile genes in smooth muscle cells and is required for closure of the ductus arteriosus in mice. J Clin Invest. 118:515-525

Ihida-Stansbury, K.; McKean, D.M.; Gebb, S.A.; Martin, J.F.; Stevens, T.; Nemenoff, R. et al. (2004) Paired-related homeobox gene Prx1 is required for pulmonary vascular development. Circ Res. 94:1507-1514

Jain, M.K.; Fujita, K.P.; Hsieh, C.M.; Endege, W.O.; Sibinga, N.E.; Yet, S.F. et al. (1996) Molecular cloning and characterization of SmLIM, a developmentally regulated LIM protein preferentially expressed in aortic smooth muscle cells. J Biol Chem. 271:10194-10199

Jones, F.S.; Meech, R.; Edelman, D.B.; Oakey, R.J. \& Jones, P.L. (2001) Prx1 controls vascular smooth muscle cell proliferation and tenascin-C expression and is upregulated with Prx2 in pulmonary vascular disease. Circ Res. 89:131-138

Kawai-Kowase, K.; Kumar, M.S.; Hoofnagle, M.H.; Yoshida, T. \& Owens, G.K. (2005) PIAS1 activates the expression of smooth muscle cell differentiation marker genes by interacting with serum response factor and class I basic helix-loop-helix proteins. Mol Cell Biol. 25:8009-8023

Kawai-Kowase, K.; Ohshima, T.; Matsui, H.; Tanaka, T.; Shimizu, T.; Iso, T. et al. (2009) PIAS1 mediates TGF $\beta$-induced SM $\alpha$-actin gene expression through inhibition of KLF4 function-expression by protein sumoylation. Arterioscler Thromb Vasc Biol. 29:99-106 
Kumar, M.S.; Hendrix, J.A.; Johnson, A.D. \& Owens, G.K. (2003) Smooth muscle $\alpha$-actin gene requires two E-boxes for proper expression in vivo and is a target of class I basic helix-loop-helix proteins. Circ Res. 92:840-847

Landerholm, T.E.; Dong, X.R.; Lu, J.; Belaguli, N.S.; Schwartz, R.J. \& Majesky, M.W. (1999) A role for serum response factor in coronary smooth muscle differentiation from proepicardial cells. Development. 126:2053-2062

Li, J.; Zhu, X.; Chen, M.; Cheng, L.; Zhou, D.; Lu, M.M. et al. (2005) Myocardin-related transcription factor $\mathrm{B}$ is required in cardiac neural crest for smooth muscle differentiation and cardiovascular development. Proc Natl Acad Sci USA. 102:89168921

Li, L.; Miano, J.M.; Mercer, B. \& Olson, E.N. (1996) Expression of the SM22 $\alpha$ promoter in transgenic mice provides evidence for distinct transcriptional regulatory programs in vascular and visceral smooth muscle cells. J Cell Biol. 132: 849-859

Li, L.; Liu, Z.; Mercer, B.; Overbeek, P. \& Olson, E.N. (1997) Evidence for serum response factor-mediated regulatory networks governing SM22 $\alpha$ transcription in smooth, skeletal, and cardiac muscle cells. Dev Biol. 187:311-321

Li, S.; Wang, D.Z.; Wang, Z.; Richardson, J.A. \& Olson, E.N. (2003) The serum response factor coactivator myocardin is required for vascular smooth muscle development. Proc Natl Acad Sci USA. 100:9366-9370

Li, S.; Chang, S.; Qi, X.; Richardson, J.A. \& Olson, E.N. (2006) Requirement of a myocardinrelated transcription factor for development of mammary myoepithelial cells. Mol Cell Biol. 26:5797-5808

Lilly, B.; Clark, K.A.; Yoshigi, M.; Pronovost, S.; Wu, M.L.; Periasamy, M. et al. (2010) Loss of the serum response factor cofactor, cysteine-rich protein 1, attenuates neointima formation in the mouse. Arterioscler Thromb Vasc Biol. 30:694-701

Liu, Y.; Sinha, S. \& Owens, G. (2003) A transforming growth factor- $\beta$ control element required for SM $\alpha$-actin expression in vivo also partially mediates GKLF-dependent transcriptional repression. J Biol Chem. 278:48004-48011

Liu, Y.; Sinha, S.; McDonald, O.G.; Shang, Y.; Hoofnagle, M.H. \& Owens, G.K. (2005) Kruppel-like factor 4 abrogates myocardin-induced activation of smooth muscle gene expression. J Biol Chem. 280:9719-9727

Long, X.; Bell, R.D.; Gerthoffer, W.T.; Zlokovic, B.V. \& Miano, J.M. (2008) Myocardin is sufficient for a smooth muscle-like contractile phenotype. Arterioscler Thromb Vasc Biol. 28:1505-1510

Mack, C.P. \& Owens, G.K. (1999) Regulation of SM $\alpha$-actin expression in vivo is dependent on CArG elements within the $5^{\prime}$ and first intron promoter regions. Circ Res. 84:852-861

Madsen, C.S.; Regan, C.P.; Hungerford, J.E.; White, S.L.; Manabe, I. \& Owens, G.K. (1998) Smooth muscle-specific expression of the smooth muscle myosin heavy chain gene in transgenic mice requires 5'-flanking and first intronic DNA sequence. Circ Res. 82:908-917

Manabe, I. \& Owens, G.K. (2001a) CArG elements control smooth muscle subtype-specific expression of smooth muscle myosin in vivo. J Clin Invest. 107: 823-834

Manabe, I. \& Owens, G.K. (2001b) Recruitment of serum response factor and hyperacetylation of histones at smooth muscle-specific regulatory regions during differentiation of a novel P19-derived in vitro smooth muscle differentiation system. Circ Res. 88:1127-1134 
Mano, T.; Luo, Z.; Malendowicz, S.L.; Evans, T. \& Walsh, K. (1999) Reversal of GATA-6 downregulation promotes smooth muscle differentiation and inhibits intimal hyperplasia in balloon-injured rat carotid artery. Circ Res. 84:647-654

Martin, J.F.; Bradley, A. \& Olson, E.N. (1995) The paired-like homeo box gene MHox is required for early events of skeletogenesis in multiple lineages. Gene Dev. 9:12371249

Miano, J.M.; Carlson, M.J.; Spencer, J.A. \& Misra, R.P. (2000) Serum response factordependent regulation of the smooth muscle calponin gene. J Biol Chem. 275:98149822

Miano, J.M. (2003) Serum response factor: toggling between disparate programs of gene expression. J Mol Cell Cardiol. 35:577-593

Miano, J.M.; Ramanan, N.; Georger, M.A.; de Mesy Bentley, K.L.; Emerson, R.L.; Balza, R.O. et al. (2004) Restricted inactivation of serum response factor to the cardiovascular system. Proc Natl Acad Sci USA. 101:17132-17137

Miralles, F.; Posern, G.; Zaromytidou, A.I. \& Treisman, R. (2003) Actin dynamics control SRF activity by regulation of its coactivator MAL. Cell. 113:329-342

Molkentin, J.D. (2000) The zinc finger-containing transcription factors GATA-4, -5, and -6: ubiquitously expressed regulators of tissue-specific gene expression. J Biol Chem. 275:38949-38952

Morrisey, E.E.; Ip, H.S.; Lu, M.M. \& Parmacek, M.S. (1996) GATA-6: a zinc finger transcription factor that is expressed in multiple cell lineages derived from lateral mesoderm. Dev Biol. 177:309-322

Morrisey, E.E.; Tang, Z.; Sigrist, K.; Lu, M.M.; Jiang, F.; Ip, H.S. et al. (1998) GATA6 regulates HNF4 and is required for differentiation of visceral endoderm in the mouse embryo. Gene Dev. 12:3579-3590

Nakamura, S.; Hayashi, K.; Iwasaki, K.; Fujioka, T.; Egusa, H., Yatani, H. et al. (2010) Nuclear import mechanism for myocardin family members and their correlation with vascular smooth muscle cell phenotype. J Biol Chem. 285:37314-37323

Nishida, W.; Nakamura, M.; Mori, S.; Takahashi, M.; Ohkawa, Y.; Tadokoro, S. et al. (2002) A triad of serum response factor and the GATA and NK families governs the transcription of smooth and cardiac muscle genes. J Biol Chem. 277:7308-7317

Norman, C.; Runswick, M.; Pollock, R. \& Treisman, R. (1988) Isolation and properties of cDNA clones encoding SRF, a transcription factor that binds to the $c$-fos serum response element. Cell. 55:989-1003

Oh, J.; Richardson, J.A. \& Olson, E.N. (2005) Requirement of myocardin-related transcription factor-B for remodeling of branchial arch arteries and smooth muscle differentiation. Proc Natl Acad Sci USA. 102:15122-15127

Owens, G.K. \& Thompson, M.M. (1986) Developmental changes in isoactin expression in rat aortic smooth muscle cells in vivo: relationship between growth and cytodifferentiation. J Biol Chem. 261:13373-13380

Owens, G.K. (1995) Regulation of differentiation of vascular smooth muscle cells. Physiol Rev. 75:487-517

Owens, G.K.; Kumar, M.S. \& Wamhoff, B.R. (2004) Molecular regulation of vascular smooth muscle cell differentiation in development and disease. Physiol Rev. 84:767-801

Oyama, Y.; Kawai-Kowase, K.; Sekiguchi, K.; Sato, M.; Sato, H.; Yamazaki, M. et al. (2004) Homeobox protein Hex facilitates serum responsive factor-mediated activation of the SM22 $\alpha$ gene transcription in embryonic fibroblasts. Arterioscler Thromb Vasc Biol. 24:1602-1607 
Philippar, U.; Schratt, G.; Dieterich, C.; Müller, J.M.; Galgóczy, P.; Engel, F.B. et al. (2004) The SRF target gene Fhl2 antagonizes RhoA/MAL-dependent activation of SRF. Mol Cell. 16:867-880

Qiu, P. \& Li, L. (2002) Histone acetylation and recruitment of serum responsive factor and CREB-binding protein onto SM22 promoter during SM22 gene expression. Circ Res. 90:858-865

Sasazuki, T.; Sawada, T.; Sakon, S.; Kitamura, T.; Kishi, T.; Okazaki, T. et al. (2002) Identification of a novel transcriptional activator, BSAC, by a functional cloning to inhibit tumor necrosis factor-induced cell death. J Biol Chem. 277:28853-28860

Schmeichel, K.L. \& Beckerle, M.C. (1994) The LIM domain is a modular protein-binding interface. Cell. 79:211-219

Schmidt, D. \& Müller, S. (2002) Members of the PIAS family act as SUMO ligases for c-Jun and p53 and repress p53 activity. Proc Natl Acad Sci USA. 99:2872-2877

Sekiguchi, K.; Kurabayashi, M.; Oyama, Y.; Aihara, Y.; Tanaka, T.; Sakamoto, H. et al. (2001) Homeobox protein Hex induces SMemb/nonmuscle myosin heavy chain-B gene expression through the cAMP-responsive element. Circ Res. 88:52-58

Selvaraj, A. \& Prywes, R. (2003) Megakaryoblastic leukemia-1/2, a transcriptional coactivator of serum response factor, is required for skeletal myogenic differentiation. J Biol Chem. 278:41977-41987

Shang, Y.; Yoshida, T.; Amendt, B.A.; Martin, J.F. \& Owens, G.K. (2008) Pitx2 is functionally important in the early stages of vascular smooth muscle cell differentiation. J Cell Biol. 181:461-473

Shaw, P.E.; Schröter, H. \& Nordheim, A. (1989) The ability of a ternary complex to form over the serum response element correlates with serum inducibility of the human $c$ fos promoter. Cell. 56:563-572

Shimizu, R.T.; Blank, R.S.; Jervis, R.; Lawrenz-Smith, S.C. \& Owens, G.K. (1995) The smooth muscle $\alpha$-actin gene promoter is differentially regulated in smooth muscle versus non-smooth muscle cells. J Biol Chem. 270:7631-7643

Sun, Y.; Boyd, K.; Xu, W.; Ma, J.; Jackson, C.W.; Fu, A. et al. (2006) Acute myeloid leukemiaassociated Mkl1 (Mrtf-a) is a key regulator of mammary gland function. Mol Cell Biol. 26:5809-5826

Sundberg-Smith, L.J.; DiMichele, L.A.; Sayers, R.L.; Mack, C.P. \& Taylor, J.M. (2008) The LIM protein leupaxin is enriched in smooth muscle and functions as an serum response factor cofactor to induce smooth muscle cell gene transcription. Circ Res. 102:1502-1511

Thomas, P.Q.; Brown, A. \& Beddington, R.S. (1998) Hex: a homeobox gene revealing periimplantation asymmetry in the mouse embryo and an early transient marker of endothelial cell precursors. Development. 125:85-94

Treisman, R.; Marais, R. \& Wynne, J. (1992) Spatial flexibility in ternary complexes between SRF and its accessory proteins. EMBO J. 11:4631-4640

Turla, M.B.; Thompson, M.M.; Corjay, M.H. \& Owens, G.K. (1991) Mechanisms of angiotensin II- and arginine vasopressin-induced increases in protein synthesis and content in cultured rat aortic smooth muscle cells: evidence for selective increases in smooth muscle isoactin expression. Circ Res. 68:288-289

Wang, D.Z.; Chang, P.S.; Wang, Z.; Sutherland, L.; Richardson, J.A.; Small, E. et al. (2001) Activation of cardiac gene expression by myocardin, a transcriptional cofactor for serum response factor. Cell. 105:851-862 
Wang, D.Z.; Li, S.; Hockemeyer, D.; Sutherland, L.; Wang, Z.; Schratt, G. et al. (2002) Potentiation of serum response factor activity by a family of myocardin-related transcription factors. Proc Natl Acad Sci USA. 99:14855-14860

Wang, J.; Li, A.; Wang, Z.; Feng, X., Olson, E.N. \& Schwartz, R.J. (2007) Myocardin sumoylation transactivates cardiogenic genes in pluripotent 10T1/2 fibroblasts. Mol Cell Biol. 27:622-632

Wang, Z.; Wang, D.Z.; Pipes, G.C.T. \& Olson, E.N. (2003) Myocardin is a master regulator of smooth muscle gene expression. Proc Natl Acad Sci USA. 100:7129-7134

Wang, Z.; Wang, D.Z.; Hockemeyer, D.; McAnally, J.; Nordheim, A. \& Olson, E.N. (2004) Myocardin and ternary complex factors compete for SRF to control smooth muscle gene expression. Nature. 428:185-189

Wei, J.; Gorman, T.E.; Liu, X.; Ith, B.; Tseng, A.; Chen, Z. et al. (2005) Increased neointima formation in cysteine-rich protein 2-deficient mice in response to vascular injury. Circ Res. 97:1323-1331

Yang, S.H.; Vickers, E.; Brehm, A.; Kouzarides, T. \& Sharrocks, A.D. (2001) Temporal recruitment of the mSin3A-histone deacetylase corepressor complex to the ETS domain transcription factor Elk-1. Mol Cell Biol. 21:2802-2814

Yang, S.H.; Jaffray, E.; Hay, R.T. \& Sharrocks, A.D. (2003) Dynamic interplay of the SUMO and ERK pathways in regulating Elk-1 transcriptional activity. Mol Cell. 12:63-74

Yang, S.H. \& Sharrocks, A.D. (2004) SUMO promotes HDAC-mediated transcriptional repression. Mol Cell. 13:611-617

Yin, F. \& Herring, B.P. (2005) GATA-6 can act as a positive or negative regulator of smooth muscle-specific gene expression. J Biol Chem. 280:4745-4752

Yoshida, T.; Sinha, S.; Dandré, F.; Wamhoff, B.R.; Hoofnagle, M.H.; Kremer, B.E. et al. (2003) Myocardin is a key regulator of CArG-dependent transcription of multiple smooth muscle marker genes. Circ Res. 92:856-864

Yoshida, T.; Hoofnagle, M.H. \& Owens, G.K. (2004a) Myocardin and Prx1 contribute to angiotensin II-induced expression of smooth muscle $\alpha$-actin. Circ Res. 94:1075-1082

Yoshida, T.; Kawai-Kowase, K. \& Owens, G.K. (2004b) Forced expression of myocardin is not sufficient for induction of smooth muscle differentiation in multipotential embryonic cells. Arterioscler Thromb Vasc Biol. 24:1596-1601

Yoshida, T. \& Owens, G.K. (2005) Molecular determinants of vascular smooth muscle cell diversity. Circ Res. 96:280-291

Yoshida, T.; Gan, Q.; Shang, Y. \& Owens, G.K. (2007) Platelet-derived growth factor-BB represses smooth muscle cell marker genes via changes in binding of MKL factors and histone deacetylases to their promoters. Am J Physiol Cell Physiol. 292:C886C895

Yoshida, T.; Gan, Q. \& Owens, G.K. (2008a) Krüppel-like factor 4, Elk-1, and histone deacetylases cooperatively suppress smooth muscle cell differentiation markers in response to oxidized phospholipids. Am J Physiol Cell Physiol. 295:C1175-C1182

Yoshida, T.; Kaestner, K.H. \& Owens, G.K. (2008b) Conditional deletion of Krüppel-like factor 4 delays downregulation of smooth muscle cell differentiation markers but accelerates neointimal formation following vascular injury. Circ Res. 102:1548-1557

Zhou, J.; Hu, G. \& Herring, B.P. (2005) Smooth muscle-specific genes are differentially sensitive to inhibition by Elk-1. Mol Cell Biol. 25:9874-9885 


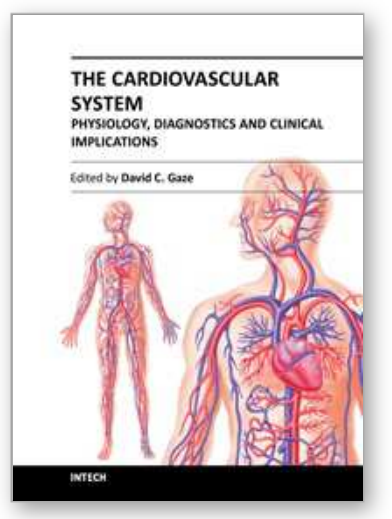

\author{
The Cardiovascular System - Physiology, Diagnostics and Clinical \\ Implications \\ Edited by Dr. David Gaze
}

ISBN 978-953-51-0534-3

Hard cover, 478 pages

Publisher InTech

Published online 25, April, 2012

Published in print edition April, 2012

The cardiovascular system includes the heart located centrally in the thorax and the vessels of the body which carry blood. The cardiovascular (or circulatory) system supplies oxygen from inspired air, via the lungs to the tissues around the body. It is also responsible for the removal of the waste product, carbon dioxide via air expired from the lungs. The cardiovascular system also transports nutrients such as electrolytes, amino acids, enzymes, hormones which are integral to cellular respiration, metabolism and immunity. This book is not meant to be an all encompassing text on cardiovascular physiology and pathology rather a selection of chapters from experts in the field who describe recent advances in basic and clinical sciences. As such, the text is divided into three main sections: Cardiovascular Physiology, Cardiovascular Diagnostics and lastly, Clinical Impact of Cardiovascular Physiology and Pathophysiology.

\title{
How to reference
}

In order to correctly reference this scholarly work, feel free to copy and paste the following:

Tadashi Yoshida (2012). Molecular Control of Smooth Muscle Cell Differentiation Marker Genes by Serum Response Factor and Its Interacting Proteins, The Cardiovascular System - Physiology, Diagnostics and Clinical Implications, Dr. David Gaze (Ed.), ISBN: 978-953-51-0534-3, InTech, Available from: http://www.intechopen.com/books/the-cardiovascular-system-physiology-diagnostics-and-clinicalimplications/molecular-control-of-smooth-muscle-cell-differentiation-marker-genes-by-serum-response-factorand-it

\section{INTECH}

open science | open minds

\section{InTech Europe}

University Campus STeP Ri

Slavka Krautzeka 83/A

51000 Rijeka, Croatia

Phone: +385 (51) 770447

Fax: +385 (51) 686166

www.intechopen.com

\section{InTech China}

Unit 405, Office Block, Hotel Equatorial Shanghai

No.65, Yan An Road (West), Shanghai, 200040, China

中国上海市延安西路65号上海国际贵都大饭店办公楼 405 单元

Phone: +86-21-62489820

Fax: $+86-21-62489821$ 
(C) 2012 The Author(s). Licensee IntechOpen. This is an open access article distributed under the terms of the Creative Commons Attribution 3.0 License, which permits unrestricted use, distribution, and reproduction in any medium, provided the original work is properly cited. 